\title{
Improved Solid Decomposition Algorithms for the CAD-to-MC Conversion Tool McCad
}

\author{
L. Lu, Y. Qiu, U. Fischer \\ lei.lu@kit.edu \\ Institute for Neutron Physics and Reactor Technology, Karlsruhe Institute for Technology (KIT). \\ Hermann-von-Helmholtz-Platz 176344 Eggenstein-Leopoldshafen,Germany
}

\section{- Introduction}

$\mathrm{McCad}$ is a geometry conversion tool to enable the automatic conversion from CAD models into the Monte Carlo (MC) geometries(MCNP, Tripoli, Geant4 ). McCad is open source and available under a GPL (General Public License) type license agreement.

CAD-MC conversion = Brep (Boundary representation)-CSG (Constructive Solid Geometry) conversion.

Step 1. Decomposition

Step 2. Void space generation

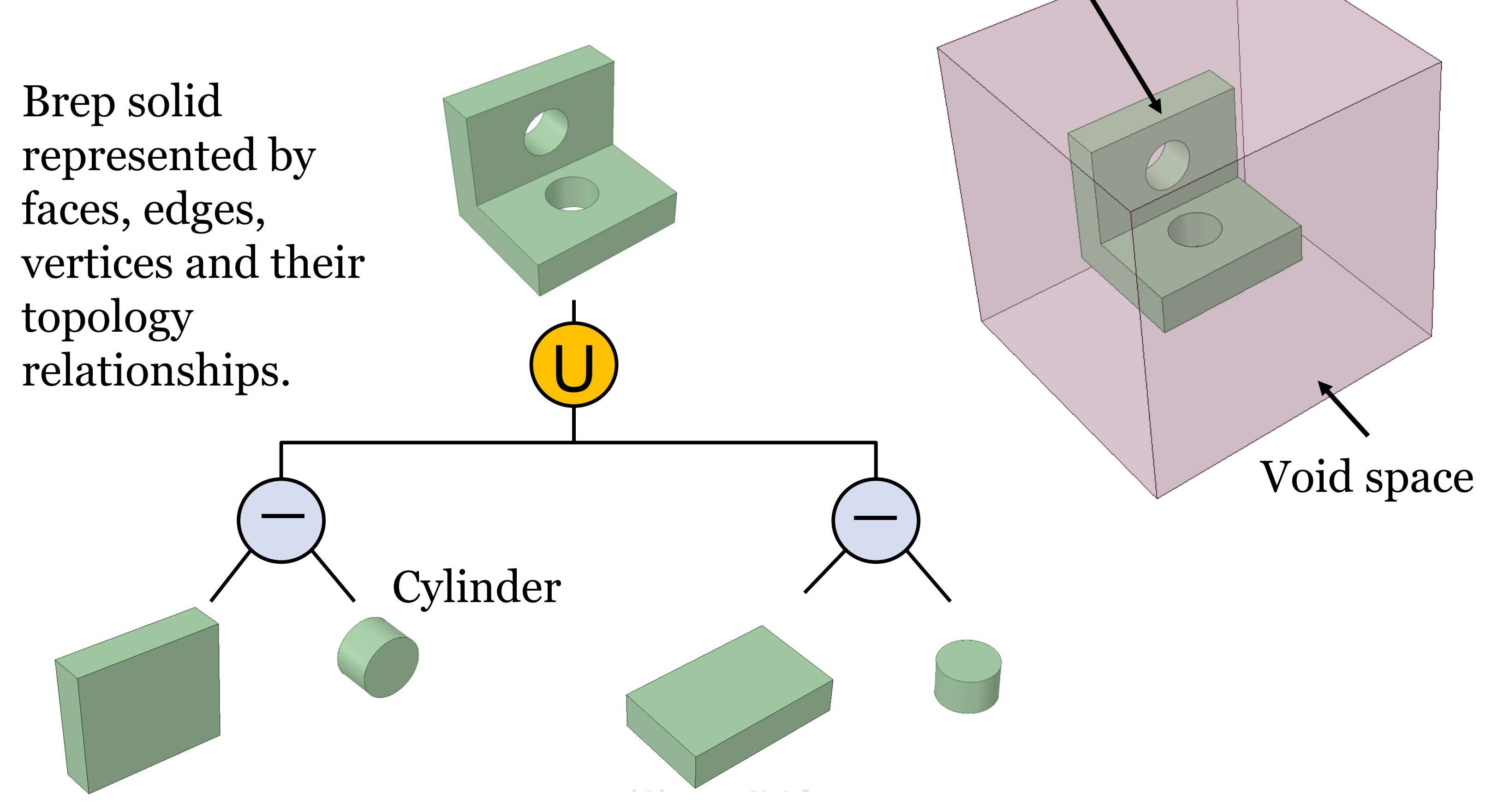

Intersection of 6

half-spaces of plane

Problem: Last version of McCad provides already well developed and validated void space generation function. Manually decomposed models can be converted into MC input files. Automatic decomposition of a CAD model is still a challenging task, mainly because the graphic kernel OpenCascade employed by McCad is not very stable and robust for Boolean operations.

\section{- New Decomposition Algorithm}

\section{Objectives}

- More efficient: Apply triangles collision detecting algorithm for searching the splitting boundary surfaces, thus avoid a large number of Boolean operations and improve the efficiency and stability.

- More robust: Add assisted splitting surfaces for separating the curved surfaces, avoid the generation of disqualified solids and subsequent Boolean operation errors. e.g., sheets and fragments.

- More intelligent: On the basis of feature recognition techniques, a sorting algorithm is developed for assigning different weights to the splitting surfaces. With the sorted surfaces, the decomposition result can be simpler and more regular, and fewer splitting processes are required.

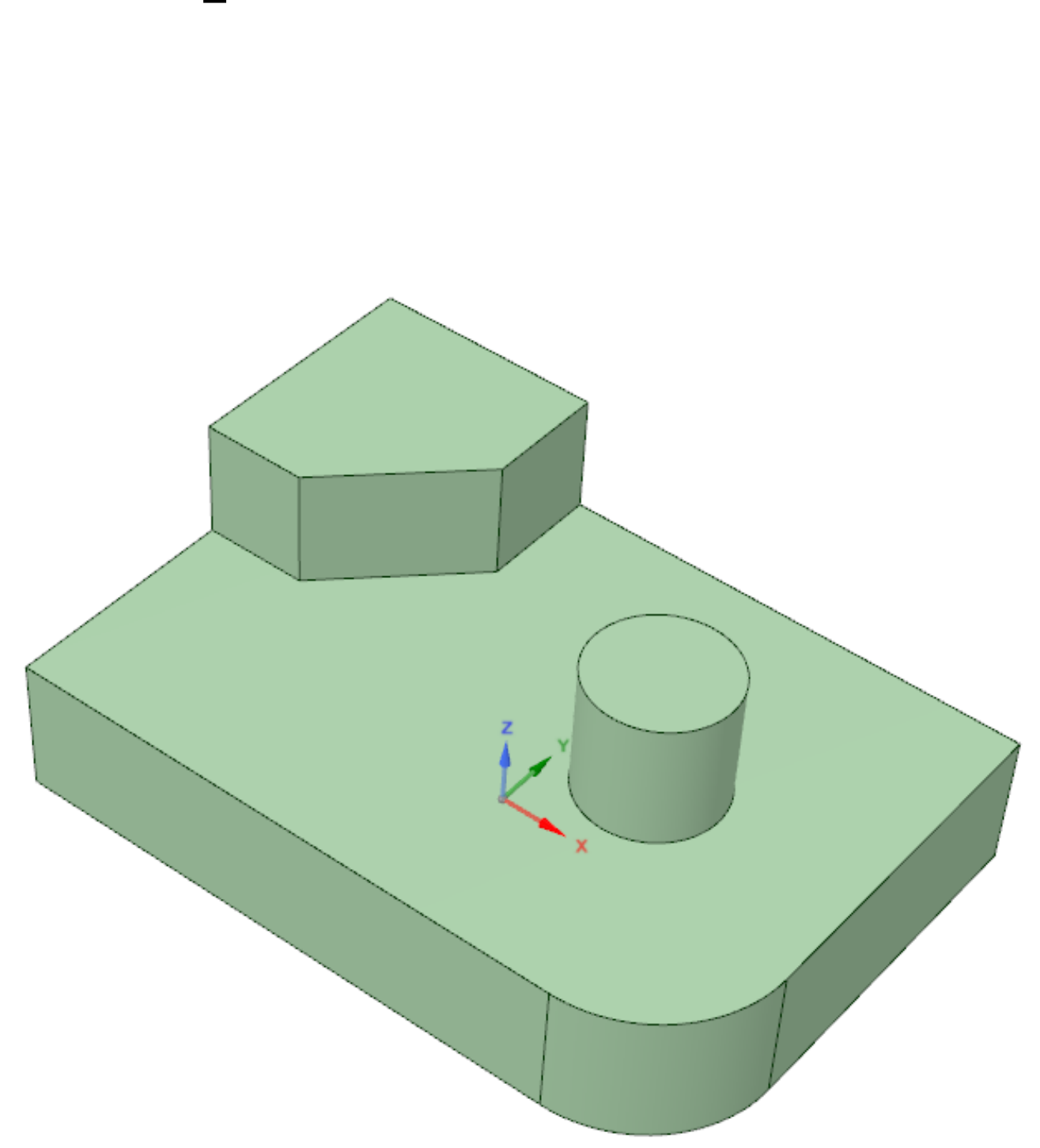

Original CAD model

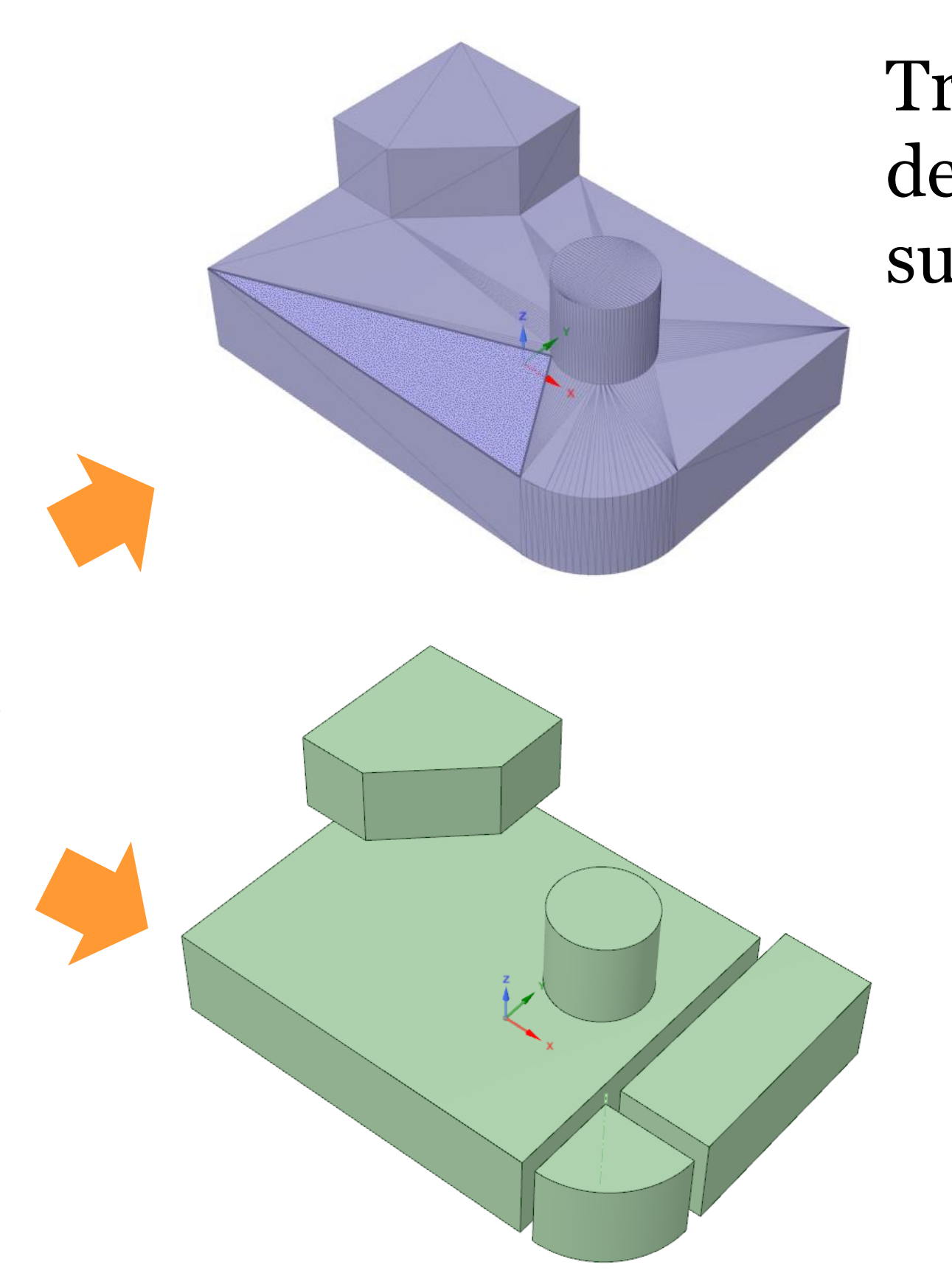

Optimal decomposition result with optimized splitting surfaces
Triangle model for detecting the splitting surfaces

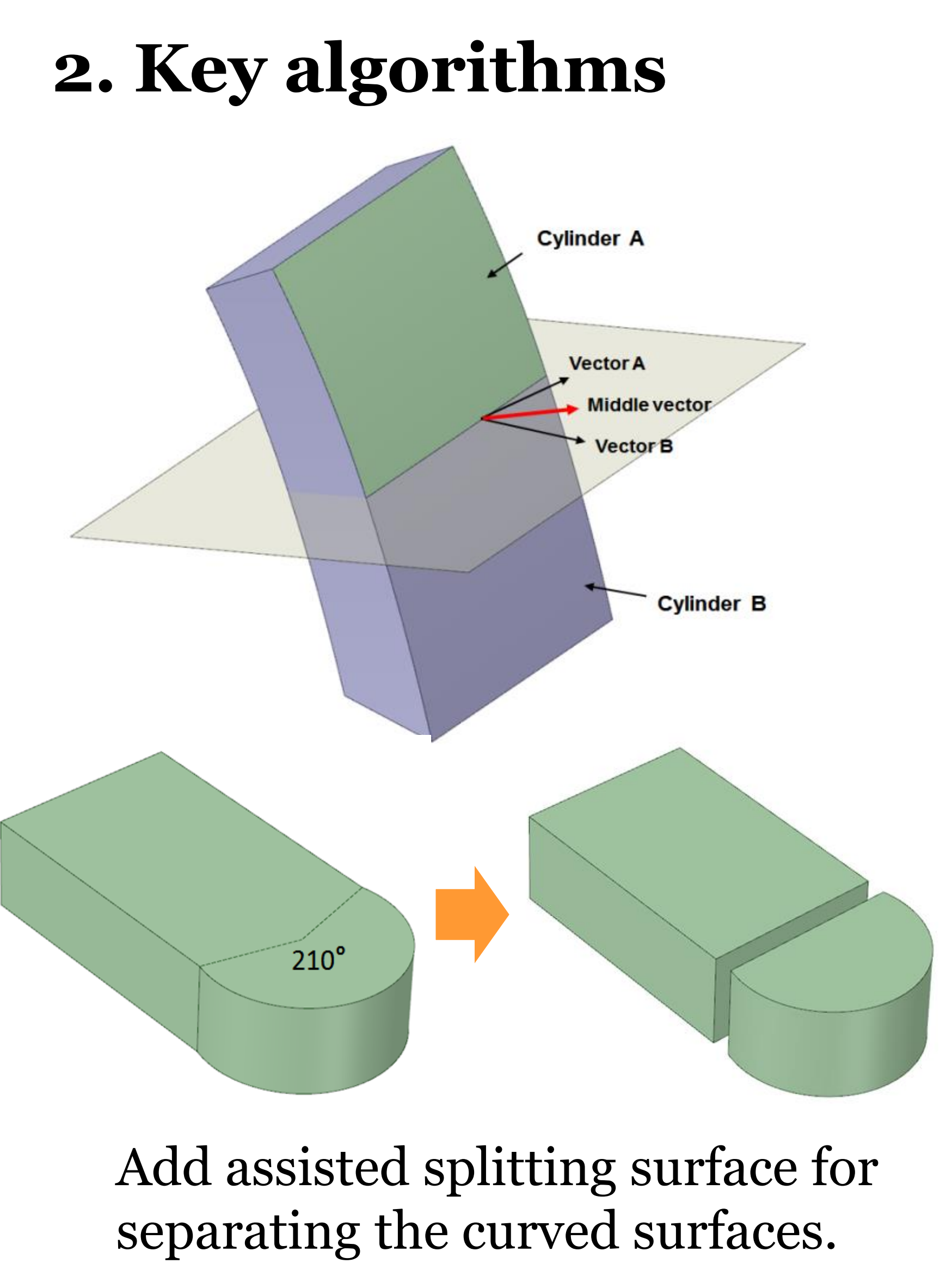

3. Workflow

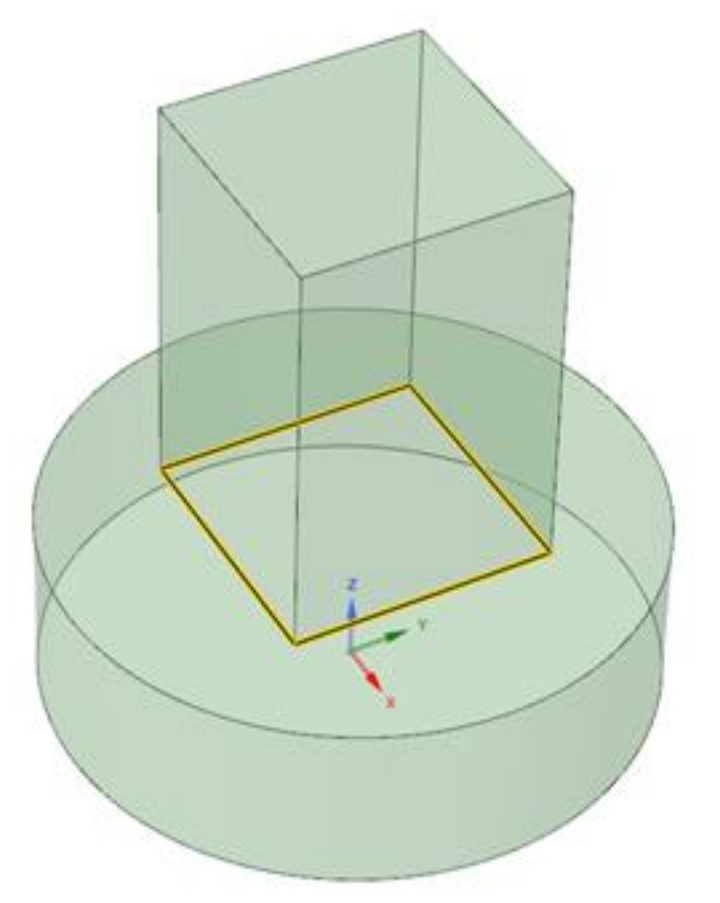

Surfaces with inner loops that have priority to be splitting surfaces

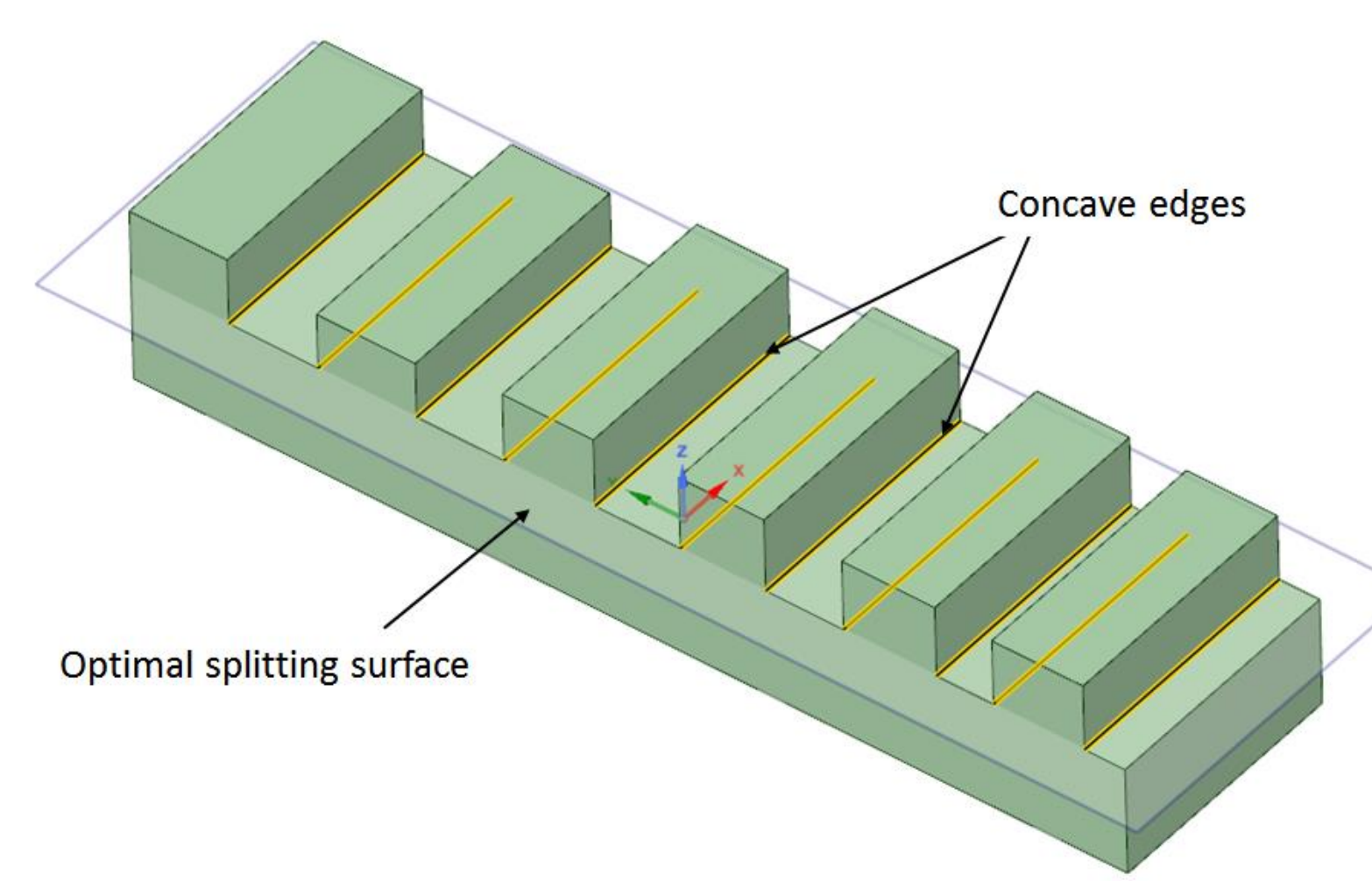

The number of concave edges through which the splitting surface passes is an important criterion for priority evaluation.

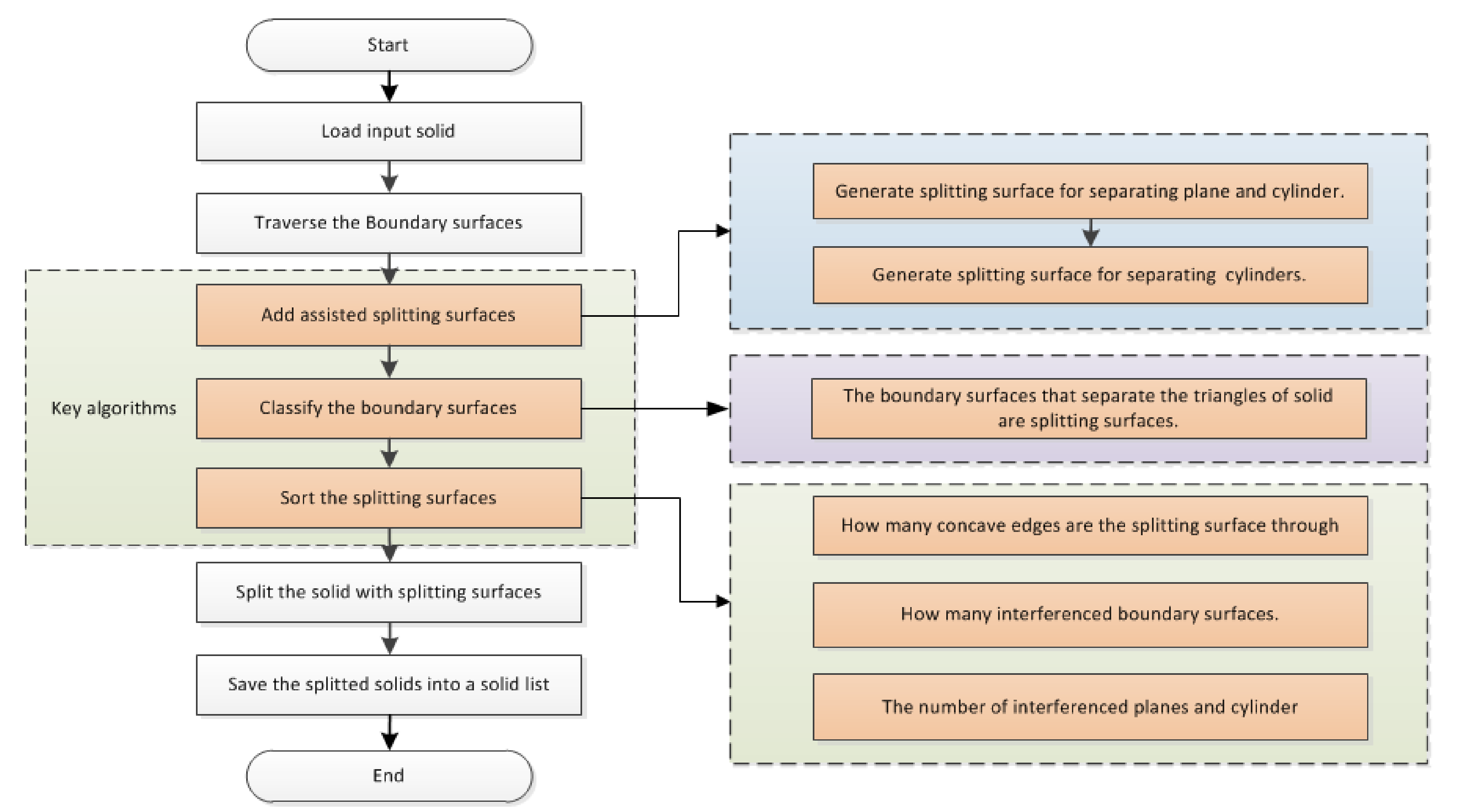

- Validations
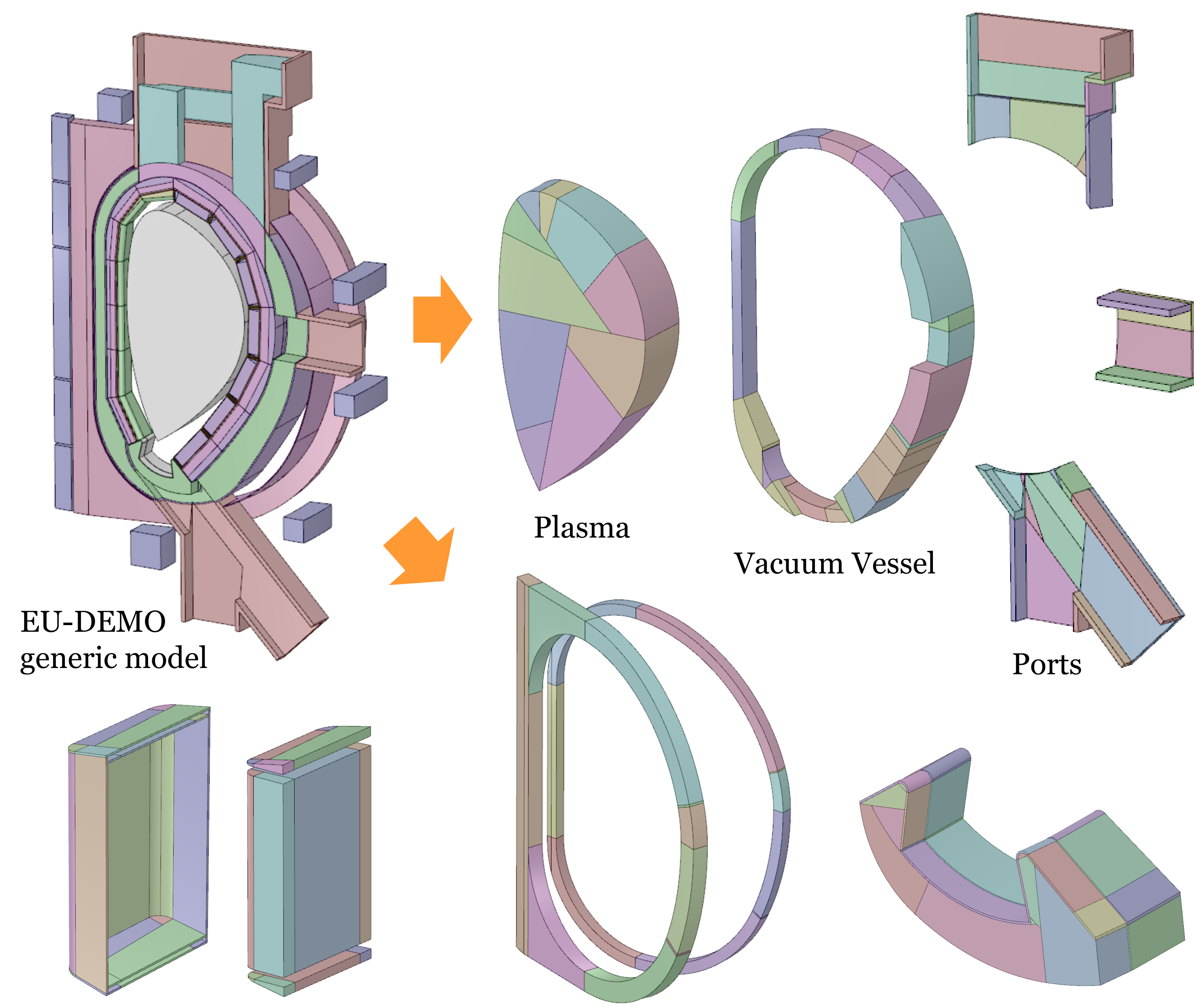

Blanket Module 